\title{
Accurate ion beam analysis in the presence of surface roughness
}

\author{
S.L. Molodtsov ${ }^{1}$, A.F. Gurbich ${ }^{1}$, C. Jeynes ${ }^{2}$ \\ ${ }^{1}$ Institute of Physics and Power Engineering, Obninsk, Russian Federation \\ ${ }^{2}$ University of Surrey Ion Beam Centre, Guildford, England
}

Corresponding author:

\author{
A.F.Gurbich \\ Institute of Physics and Power Engineering \\ Bondarenko sq. 1 \\ 249033, Obninsk \\ Russian Federation \\ E-mail: gurbich@ippe.ru
}

\section{PACS Keywords:}

02.70.Uu Applications of Monte Carlo methods,

68.35.Ct Interface structure and roughness,

68.35.Dv Composition, segregation; defects and impurities,

68.49.-h Surface characterization by particle-surface scattering,

89.20.Bb Industrial and technological research and development

This article was published as:

J. Phys. D-Appl.Phys. 41(20), Article number 205303 (7 pp), 21 Oct 2008;

http://dx.doi.org/10.1088/0022-3727/41/20/205303 


\section{Abstract}

Ion beam analysis (IBA) is a powerful materials characterization technique with very wide applicability. However, despite the fact that most natural and many industrial samples are rough, there is currently no way to correctly take severe roughness into account when processing the IBA spectra from rough samples, without resorting to Monte Carlo calculations which are too slow for routine use. In the present work we demonstrate a new approach which parameterises a Monte Carlo calculation so that the analytical codes can rapidly calculate the effect of asperities for a wide variety of rough surfaces. We successfully apply this method to real samples. This new analytical algorithm allows us to overcome the long standing problem of the correct depth profiling of these common samples, hence dramatically increasing the power of the IBA technique. It also allows us to extract physically meaningful roughness parameters on samples whose roughness cannot be easily measured directly.

Keywords: Roughness, RBS, EBS, ERD, PIXE, Monte Carlo simulation 


\section{Introduction}

Much effort has been applied to the problem of the influence of target surface roughness on experimental particle scattering spectra (including Rutherford backscattering, RBS). However, it is not currently feasible to routinely model the effects of severe roughness on IBA spectra, where "severe" here refers to the probing beam exiting and reentering the sample as it passes through the surface asperities. For example, recent IBA work on rough samples obtained from nuclear fusion reactors [1], PECVD ZnO films [2], or LPCVD polycrystalline Si films [3] has either used scattering conditions to minimise severe roughness effects, or has simply ignored them. Even the moderate roughness of implanted insulators [4] would be expected to give noticeable effects for RBS, effects which would be magnified at the glancing geometries used for elastic recoil detection (ERD) of the light elements. In an ion beam analysis of a painting where the effect of roughness on the particle scattering spectra was artificially fitted, the particle induced Xray emission (PIXE) absorption for the pigment, uncorrected for roughness, may be in error by a factor of two [5]. We will show that not only can these effects on the IBA spectra be easily modelled without prior knowledge of the sample roughness, but that information about this roughness is extracted from the spectra by this modelling.

Fitting IBA spectra more precisely is becoming more important as the accuracy available from the technique increases. RBS spectra have historically been hard to analyse for all but rather simple samples since the the energy of a scattered ion is determined both by the mass of the scattering nucleus and its depth, making the extraction of the depth profile formally an ill-posed problem. But this ill-posed problem has had recent solutions both using maximum entropy techniques $[6,7]$ and using the simulated annealing algorithm [8]. With these new methods IBA is now routinely applied to accurately depth profile any 
smooth sample presented: but most real samples are not smooth!

Roughness can produce visible effects of two kinds: (i) Firstly, for a thin layer the trailing (low energy) edge of the corresponding backscattering spectrum is spread because the surface and interface roughness results in variations of the layer thickness across the beam spot area. This effect was investigated $[9,10,11,12,13]$ and is accurately accounted for in the "new generation" codes widely used for spectral simulation [14].

(ii) Secondly, for any target with severe roughness, the leading (high energy) edge of the spectrum is spread when the outgoing particle scattered from the rough surface may traverse asperities (Fig.1), exiting and re-entering the sample material at least once. We shall refer to this effect as "secondary crossings". For all analyses where the incident beam is not normal to the surface the effect of secondary crossings have to be taken into account for both the incident and exit particles. This effect has been addressed by various authors. For some special profiles of the surface roughness it was investigated analytically $[15,16,17]$ and the statistical (Monte-Carlo) method was applied for more general cases $[18,19,20]$. Unfortunately the analytical results are applicable only for some rather artificial surfaces, whereas statistical calculations are extremely time consuming. Subdivision of the sample surface into pixels with superimposition of spectra for each pixel [21] is also hardly applicable in the case of a surface with fine structure. Consequently, the effect (ii) is not accounted for in any currently available general purpose code.

The aim of the present work is to develop an approach which will make it possible to take the effect of secondary crossings into account in a general way suitable for use in IBA codes. This is achieved by a combination of the statistical and analytical approaches using the advantages of each. 


\section{The algorithm}

We use the following algorithm in order to model the shape of a general rough

surface. A random height chosen from a Gaussian distribution of variance $\sigma^{2}$ was assigned to a regular linear grid of reference points with separation $d$. The surface profile is then created by a spline interpolation between these points. To characterize the surface peak sharpness a parameter $p$ is defined as the common logarithm of the dimensionless ratio of $\sigma$ to $d$ :

$p=\log \frac{\sigma}{d}$

For convenience we use units of $\sigma$ throughout the paper. Only those particles which cross at least one valley between peaks and again traverse the surface will contribute to the spreading effect under investigation. The relative fraction $n$ of the scattered particles which cross one or more valleys depends on the sharpness (the $p$ parameter) and on the scattering angle $\theta$.

The approach employed in the present work consisted of the following steps. First, the fraction $n$ and the distribution of the particle pathlength $x$ in the asperities for the set of surface structures characterized by various parameters $p$ were determined through grid Monte-Carlo calculations for different scattering angles $\theta$. Then the distribution of this additional exit pathlength obtained with the MC calculation was approximated by a parameterization function $f(x)$ with some free parameters $n, a, b$ (see equation (4) below) for every combination of the input parameters $p$ and $\theta$. Each of these free parameters was assumed to be a function of $p$ and $\theta$ and was parameterized by a combination of an exponential function and a quadratic one. The function used for parameterization was

$$
G(\theta, p)=K(\theta, p, q) \exp [K(\theta, p, r)]+K(\theta, p, s)
$$


where $G$ represents, in sequence, the parameters: $n, a, b ; q, r, s$ are the vectors of free parameters of approximation, and $K$ has the form

$$
K(\theta, p, v)=v_{1} \theta^{2}+v_{2} p^{2}+v_{3} \theta p+v_{4} \theta+v_{5} p+v_{6}
$$

where $v=\left\{v_{1}, v_{2}, \ldots, v_{6}\right\}$ is a vector of free parameters and denotes $q$, $r$ or $s$ in equation (2). The free parameters of approximation were determined by the Levenberg-Marquardt algorithm.

The parameter $n$ which describes a fraction of the scattered particles which have undergone secondary crossing of surface peaks was also parameterized in the same manner. Thus a unequivocal functional dependence between the exit extra pathlength probability density function $f(x)$ and the input parameters $p$ and $\theta$ was established. The following function $f(x)$ was found to be suitable as a parameterization function:

$$
f(x)=(1-n) \delta(x)+n \frac{b^{a+1}}{\Gamma(a+1)} x^{a} \exp (-b x)
$$

where $a$ and $b(\mathrm{a}>-1$ and $\mathrm{b}>0)$ are free parameters and $\Gamma$ is the gamma function. The term containing $\delta(x)$ (the Dirac delta function) represents the part of the scattered beam which does not pass through asperities and is calculated correctly by existing codes.

The first moment of $f(x)$ gives a mean extra pathlength in the surface asperities which can be easily obtained:

$$
\langle x\rangle=\int_{0}^{\infty} x \cdot f(x) d x=n \frac{a+1}{b}
$$

Examples of Monte Carlo simulations and corresponding analytical approx- 
imations for two scattering angles are shown in Fig. 2. It is seen from the figure that the particle exit pathlength distribution significantly changes its shape depending on the scattering angle, and that the function used for the approximation adequately reproduces the distribution for different angles. The peak at $x$ close to zero corresponds to the first term in equation (4). The results obtained for the functional dependence of parameters $n, a$, and $b$ on the input parameters $p$ and $\theta$ (see equation 2) are shown in Fig. 3.

To account for the effect of secondary crossings, the uncorrected spectrum $Y_{0}(E)$ should be convoluted with the $f(x)$ function (equation 4) expressed in energy coordinates. For normal incidence the resulting backscattering spectrum $Y(E)$ is

$Y(E)=\int_{0}^{\infty} Y_{0}\left(E^{\prime}\right) \frac{f\left(X\left(E, E^{\prime}\right)\right)}{S\left(E^{\prime}\right)} d E^{\prime}$

where $S(E)$ is the stopping power and $X\left(E, E^{\prime}\right)$ is the distance passed by a particle with the initial energy $E^{\prime}$ and the final energy $E$ :

$X\left(E, E^{\prime}\right)=\int_{E}^{E^{\prime}} \frac{d \widehat{E}}{S(\widehat{E})}$

It is evident that the first term of the function $f$ in equation (4) gives in the result of integration (equation 6) the unaffected $(1-n)$ fraction of $Y_{0}(E) . S(E)$ in equation (6) is actually the stopping power in the matter of which the surface asperities consist. Thus the result of the convolution does not depend on the structure of the deeper layers of the sample. Only the composition of the asperities is significant.

An additional straggling should be also included in the resulting spectrum because of the increase of the the scattered (exit) particle pathlength due to travelling through the asperities. Strictly speaking, this correction should also be done by convolution. However since the correction is small, and taking into account the fact that the variance of the 
straggling is proportional to the pathlength in matter, it is possible to make use of the first moment of the function $f(x)$ (equation 5).

As an example, Figure 4 shows the best available calculated spectra obtained from current analytical codes (here SIMNRA [22] is used) with and without roughness. A spectrum calculated with the present approach is also shown for comparison; in this case the roughness effect lowers the average height in addition to the strong effect at the leading and trailing edges. However the areas under the spectra are equal. Further spectra calculated with various values of sharpness, scale, scattering angle and stopping power are shown in Figure 5.

Up to now, we have only considered normal beam incidence. The method is easily generalized: we need only to make an integral transform like equations (6-7) where $Y_{O}\left(E^{\prime}\right)$ is interpreted as the energy distribution function of the incident beam ions as they traverse the sample. The distribution function $Y(E)$ obtained should then be used instead of $Y_{0}\left(E^{\prime}\right)$ in the further spectrum calculations.

\section{Verifying the algorithm}

As an experimental verification of the approach we shot-blasted some tool steel sheet with (worn) $\sim 6 \mu \mathrm{m}$ pellets, giving a rough surface which was quantified on a Talystep mechanical profiler (Fig. 6) from which a roughness standard deviation of 0.95 $\mu \mathrm{m}$ was determined. RBS spectra were obtained using a proton beam from the Surrey $2 \mathrm{MV}$ Tandetron accelerator [23] of $1045 \mathrm{keV}{ }^{1} \mathrm{H}^{+}$with a detector of energy resolution $16 \mathrm{keV}$ and a scattering angle of $149^{\circ}$. The beam had a nominal diameter of $1 \mathrm{~mm}$ and was normal to the sample. The beam current was less than $5 \mathrm{nA}$ to reduce pileup effects.

Fig. 7 shows the spectra from the shot blasted and virgin samples together with a fit using the new algorithm. The following optimal values for the parameters of the surface 
roughness were found: $\sigma=1.0 \mu \mathrm{m}, p=-0.18$. The parameters of the function $f(x)$ (equation 4 ) corresponding to the scattering angle used and to the optimal $p$ value were as follows: $a=5.169, b=2.228$, and $n=0.123$. A small amount of oxygen was added to the surface layers of the sample in order to closely reproduce the measured spectrum.

Fig. 8 shows the data from Fig. 3 of Beck et al. cited above (ref.5) fitted with the new roughness algorithm. This data was taken with a $3 \mathrm{MeV}$ external proton beam (at the ALGAE facility in the Louvre Museum) of a painting from their collection. The Pb signal from the lead white base coat clearly shows the effect of severe roughness, which is well fitted using parameters $\sigma=1.0 \mu \mathrm{m}, p=-0.18$. But, as mentioned above, in the fit of Fig. 3 of ref.5 the roughness could not be modelled correctly, and the signal from the resonance in the non-Rutherford elastic backscattering (EBS) O cross-section at $2662 \mathrm{keV}$ is still clearly visible. This resonance signal shows that, as expected, the effective energy straggling of the beam at that depth cannot be calculated correctly if roughness is not taken into account. However, if the new algorithm is properly applied to the inward path as well as the outward path (as shown in Fig.8) then this straggling is indeed correctly modelled, and the fit to the data is excellent.

\section{Conclusion}

We have developed a new algorithm for the simulation of the influence in IBA spectra of the effect of severe surface roughness where the exit beam has secondary crossings of surface asperities. The algorithm is demonstrated here for RBS, but it calculates the excess pathlength variation through the asperities of the rough surface, and is therefore applicable equally to all IBA spectra. The user of the algorithm needs only analytical calculations to determine the sharpness and scale of the roughness on any particular rough surface. Moreover, only these two parameters are required to generate a 
general rough surface, and so samples can be reliably analysed whose roughness is unknown, with valid roughness parameters being obtained by the analysis.

We have shown that the algorithm can be applied to RBS spectra of very rough samples made by shot blasting, with the extracted roughness parameters being consistent with those measured, and with excellent fits to the data. We have also shown that the algorithm can be successfully applied to EBS (non-Rutherford) spectra of samples where the roughness cannot easily be measured, and that roughness parameters can be extracted from the particle backscattering data; this is the first time that spectra have been inverted to determine roughness of a surface which cannot be measured directly. Moreover, EBS allows one to probe the effective energy straggling at given depths through the use of resonances in the non-Rutherford scattering cross-sections. We have shown that the new algorithm also models this straggling correctly.

\section{Acknowledgements}

AFG is grateful to the Royal Society for supporting the short visit to Surrey (2007/R4), and CJ is grateful to EPSRC for support under EP/D032210/1. We are also grateful to Mark Browton for making the shot-blasted sample. 


\section{References}

1. I. Bizyukov, K. Krieger, N. Azarenkov, and U. v. Toussaint, Relevance of surface roughness to tungsten sputtering and carbon implantation, J. Appl. Phys. 100, 113302 (2006)

2. I. Volintiru, M. Creatore, B. J. Kniknie, C. I. M. A. Spee, and M. C. M. van de Sanden, Evolution of the electrical and structural properties during the growth of Al doped ZnO films by remote plasma-enhanced metalorganic chemical vapor deposition, J. Appl. Phys. 102, 043709 (2007)

3. P. Petrik, T. Lohner, M. Fried, L. P. Biró, N. Q. Khánh, J. Gyulai, W. Lehnert, C. Schneider, and H. Ryssel, Ellipsometric study of polycrystalline silicon films prepared by low-pressure chemical vapor deposition, J. Appl. Phys. 87, 1734 (2000)

4. M. Yedji, G.G. Ross, Effect of electric charge accumulation on the modification of surface properties by means of low energy ion implantation, J. Phys. D: Appl. Phys. 39, 4429-4435 (2006)

5. L. Beck, C. Jeynes, N.P. Barradas, Characterization of paint layers by simultaneous self-consistent fitting of RBS/PIXE spectra using simulated annealing, Nucl. Instrum. and Meth. B, 266, 1871-1874 (2008)

6. E. Edelmann, K. Arstila, J. Keinonen, Bayesian data analysis for ERDA measurements, AIP Conf. Proc. 735 (Bayesian Inference and Maximum Entropy Methods in Science and Engineering, eds. R. Fischer, R. Preuss, U. Von Toussaint), 52-59 (2004) 
7. R. Fischer, M. Mayer, W. von der Linden, V. Dose, Enhancement of the energy resolution in ion-beam experiments with the maximum-entropy method, Phys. Rev. E 55, 6667-3 (1997)

8. C. Jeynes, N.P. Barradas, P.K. Marriott, G. Boudreault, M. Jenkin, E. Wendler and R.P. Webb, Elemental thin film depth profiles by ion beam analysis using simulated annealing—a new tool, J. Phys. D: Appl. Phys. 36, R97-R126 (2003)

9. H. Metzner, M. Gossla, Th. Hahn, Rutherford backscattering spectroscopy of rough films: Theoretical considerations. Nucl. Instr. and Meth. B 124, 567-574 (1997).

10. H. Metzner, Th. Hahn, M. Gossla, J. Conrad, J.-H. Bremer, Rutherford backscattering spectroscopy of rough films: Experimental aspects. Nucl. Instr. and Meth. B 134, 249-261 (1998).

11. M. Mayer, Ion beam analysis of rough thin films. Nucl. Instr. and Meth. B 194, 177-186 (2002).

12. N.P. Barradas, Rutherford backscattering analysis of thin films and superlattices with roughness, J. Phys. D: Appl. Phys. 34, 2109-2116 (2001)

13. N.P. Barradas, Fitting of RBS data including roughness: Application to Co/Re multilayers. Nucl. Instr. and Meth. B 190, 247-251 (2002)

14. N.P. Barradas, K. Arstila, G. Battistig, M. Bianconi, N. Dytlewski, C. Jeynes, E. Kótai, G. Lulli, M. Mayer, E. Rauhala, E. Szilágyi, M. Thompson, Summary of "International Atomic Energy Agency intercomparison of ion beam analysis software". Nucl. Instr. and Meth. B 266, 1338-1342 (2008). 
15. R. Behrisch, S. Grigull, U. Kreissig, R. Grötzschel, Influence of surface roughness on measuring depth profiles and the total amount of implanted ions by RBS and ERDA. Nucl. Instr. and Meth. B 136-138, 628-632 (1998).

16. V.S. Shorin, A.N. Sosnin, The shape of the ion backscattering spectrum for a surface having sine wave relief. Nucl. Instr. and Meth. B 53, 199-201(1991).

17. Y. Itoh, T. Maeda, T. Nakajima, A. Kitamura, N. Ogivara, M. Saidoh, Simulation of RBS spectra for a surface with a periodic roughness. Nucl. Instr. and Meth. B 117, 161-169 (1996).

18. M. Wüest, P. Bochsler, Simulation of ion backscattering from rough surfaces Nucl. Instr. and Meth. B 71, 314-323 (1992).

19. T. Sajavaara, K. Arstila, A. Laakso, J. Keinonen, Effects of surface roughness on results in elastic recoil detection measurements. Nucl. Instr. and Meth. B 161-163, 235-239 (2000).

20. Z. Hajnal, E. Sziligyi, F. Paszti, G. Battistig. Channeling-like effects due to the macroscopic structure of porous silicon Nucl. Instr. and Meth. B 118 617-621 (1996)

21. I.M. Yesil, W. Assmann, H. Huber, K.E.G. Löbner, Simulation of surface roughness effects in ERDA. Nucl. Instr. and Meth. B 136-138, 623-627 (1998).

22. M. Mayer, SIMNRA, a Simulation Program for the Analysis of NRA, RBS and ERDA. AIP Conf. Proc. 475, 541-545 (1999).

23. A.Simon, C.Jeynes, R.P.Webb, R.Finnis, Z.Tabatabian, P.J.Sellin, M.B.H.Breese, D.F.Fellows, R.van den Broek, R.M.Gwilliam, The new Surrey ion beam analysis facility. Nucl. Instr. and Meth. B 219-220, 405-409 (2004) 


\section{Figure captions}

\section{Figure 1 | Schematic of rough surfaces.}

The distance $d$ characterizes the effective frequency of the roughness. The dimensionless parameter $p$ characterizes the sharpness of the asperities and is defined by eq. 1 .

Figure 2 | Numerical basis of the method.

Approximation of the statistical calculation histograms with the function $f(x)$ using $p=1$ (see text). a) Glancing scattering case $\left(\theta=100^{\circ}\right)$; b) Nearly normal scattering case $(\theta=$ $\left.170^{\circ}\right)$

\section{Figure 3 | Parameterizing the Monte Carlo calculations.}

The functional dependence determined between $n, a$, and $b$ and input parameters $p$ and $\theta$ for this model. 


\section{Figure 4| Effect of roughness on ion beam analysis spectra.}

$1 \mathrm{MeV} \mathrm{H}^{+}$Rutherford backscattering energy spectra are simulated from a flat sample (line), from a rough sample without taking secondary crossings into account (dotted line, using the SIMNRA code [ref.12]), and from a rough sample calculated correctly using the new algorithm (hashed line). The sample is a self-supporting gold layer with mean thickness $\mathrm{d}=5 \times 10^{18} \mathrm{Au}$-atoms $/ \mathrm{cm}^{2}(850 \mathrm{~nm})$, with roughness of standard deviation $\sigma=$ $5 \times 10^{17}$ atoms $/ \mathrm{cm}^{2}(85 \mathrm{~nm})$ and peak shape parameter $p=1$ (see equation 1). Incident angle $\alpha=0^{\circ}$, scattering angle $\theta=150^{\circ}$. Spectrometry system energy resolution was $15 \mathrm{keV}$.

\section{Figure 5 | Calculated Rutherford backscattering spectra from rough thick} aluminum.

Various values of the sharpness (peak shape) parameter $p$, the scale (roughness standard deviation) parameter $\sigma$, the scattering angle $\theta$ and the beam are considered. Normal beam incidence. Energy resolution of the spectrometry system is $15 \mathrm{keV}$.

Figure 6 | A mechanical profile of the shot-blasted steel surface

Obtained with a Talystep and a stylus of diameter $12.5 \mu \mathrm{m}$

\section{Figure 7 | Rutherford backscattering spectrum of shot blasted steel}

Experimental and simulated RBS spectra for a scattering angle of $149^{\circ}$ and $1045 \mathrm{keV}$ proton beam. The sharpness $p$ and the scale $\sigma$ of this rough surface are determined as $p=$ -0.18 and $\sigma=1 \mu \mathrm{m}$.

Figure 8 | 3MeV proton elastic (non-Rutherford) backscattering spectrum of painting 
(lead-white on canvas) with fit using roughness algorithm (data from Beck et al, ref.5, Fig.3).

The $\mathrm{Pb}, \mathrm{O}, \mathrm{C}, \mathrm{He}$ surface signal positions are marked. He is from the helium atmosphere in external beam apparatus. Notice that the $\mathrm{O}$ resonance at $2662 \mathrm{keV}$ whose position in the spectrum is marked " $\mathrm{R}$ " is not visible (see text)
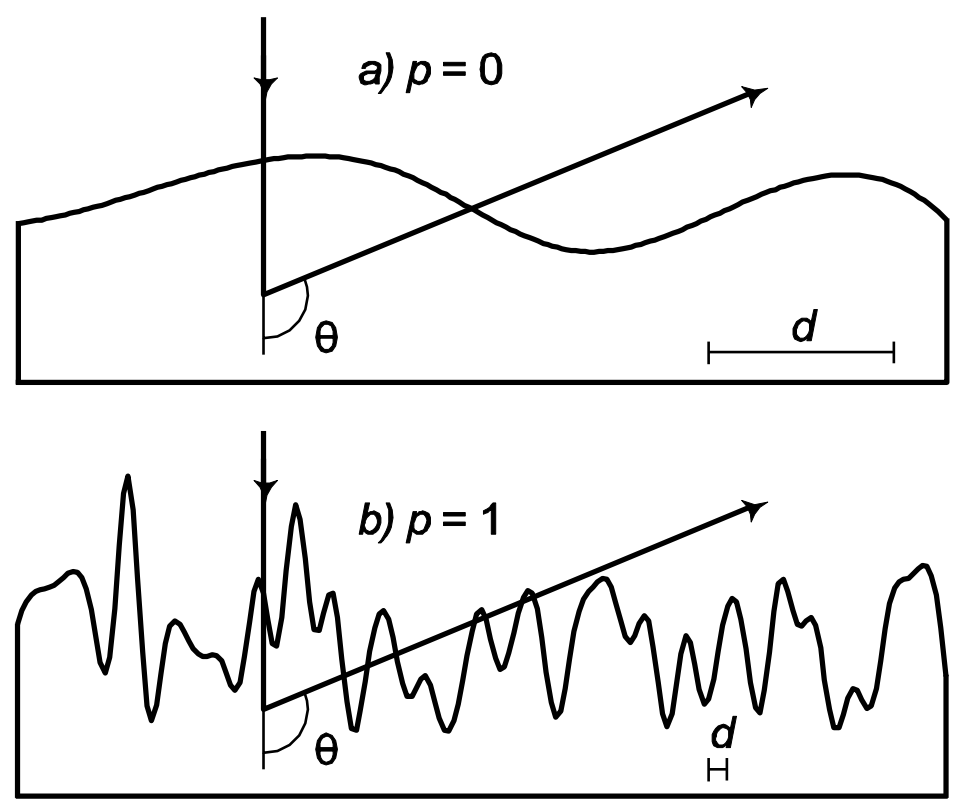

Figure 1 | Schematic of rough surfaces. 

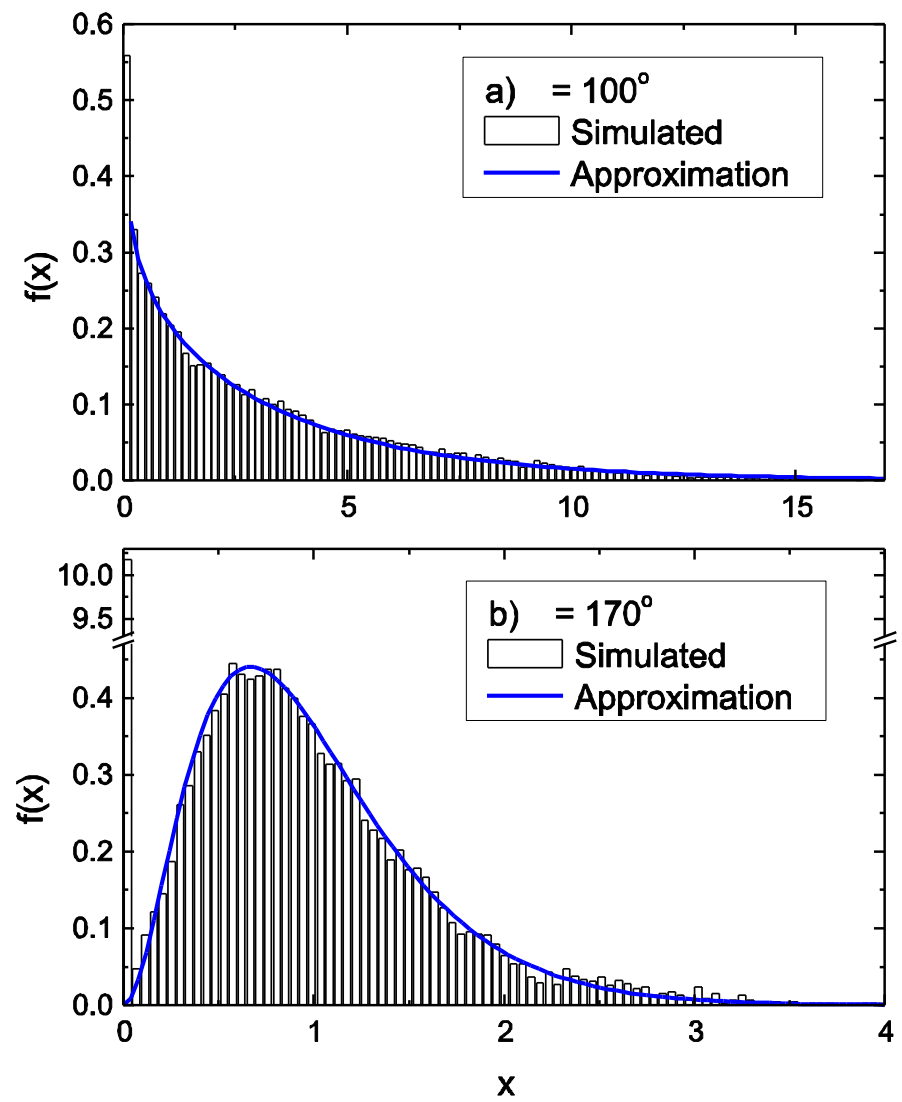

Figure 2 | Numerical basis of the method. 
Figure 3 | Parameterizing the Monte Carlo calculations. 


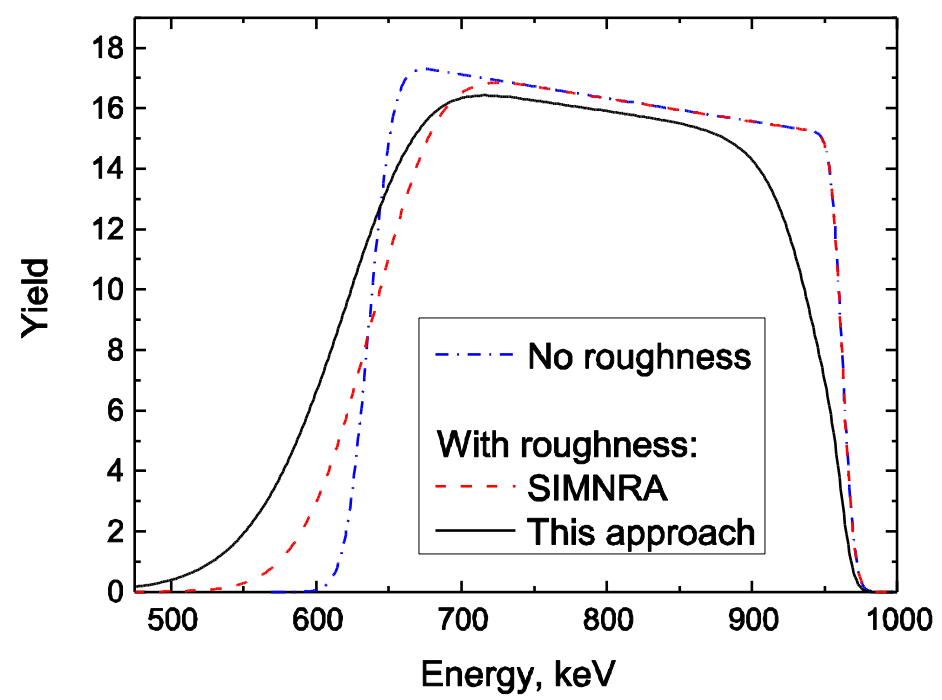

Figure 4 | Effect of roughness on ion beam analysis spectra. 

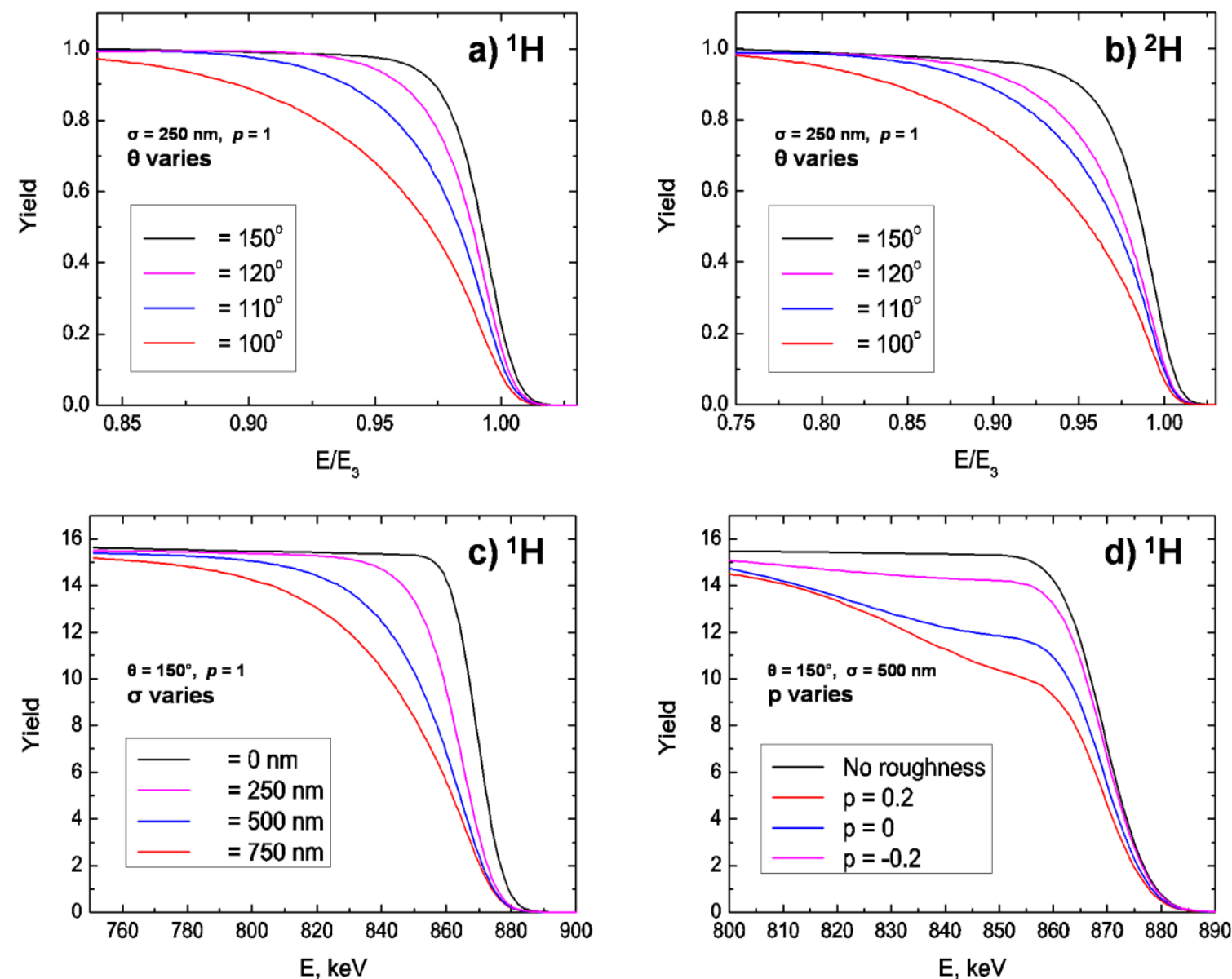

Figure 5 | Calculated Rutherford backscattering spectra from rough thick aluminum. 


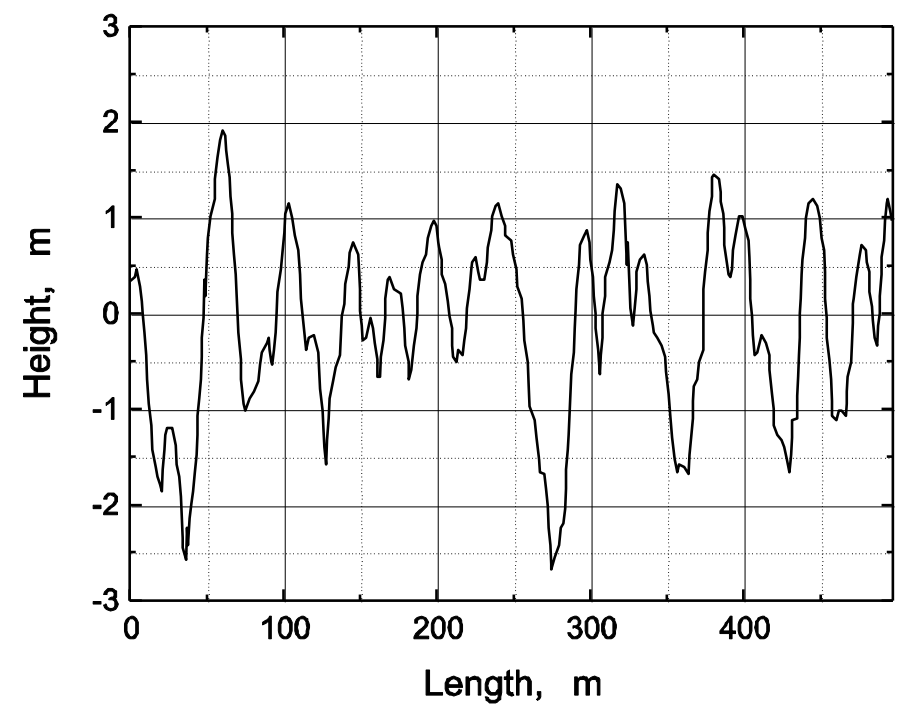

Figure 6 | A mechanical profile of the shot-blasted surface

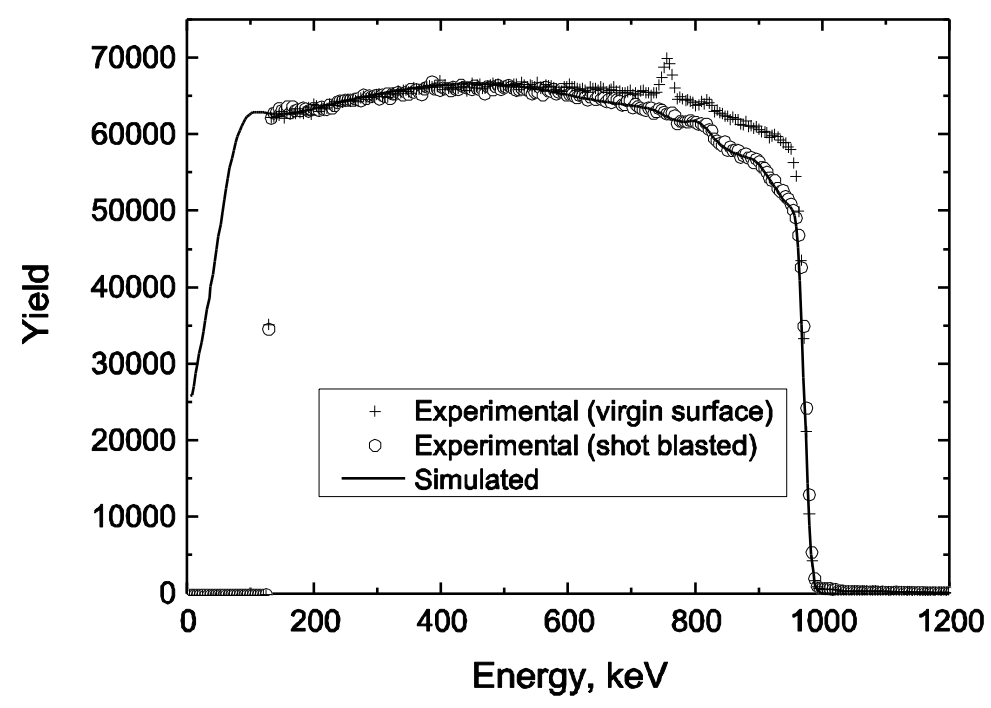

Figure 7 | Rutherford backscattering spectrum of shot blasted steel 


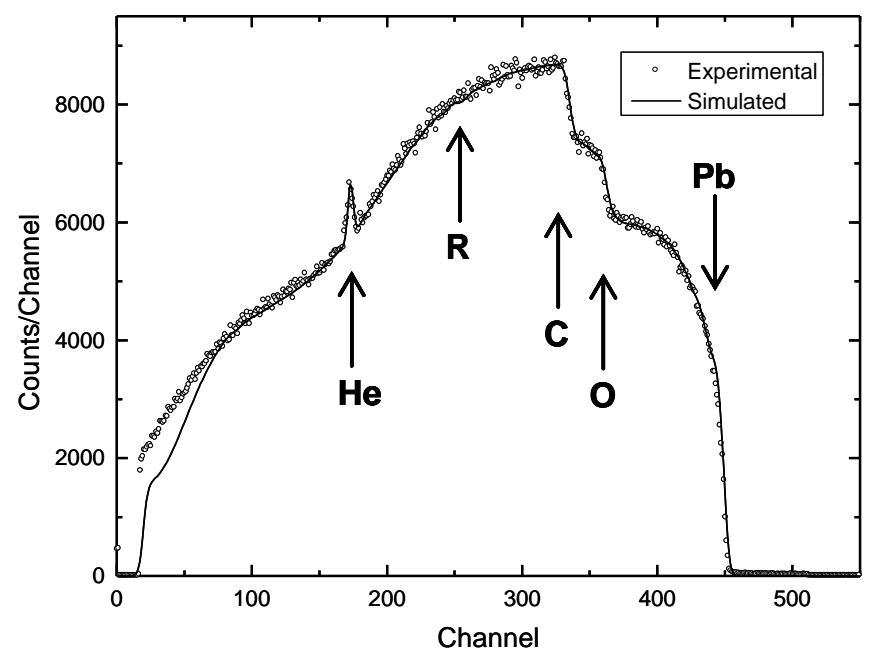

Figure 8 | 3MeV proton elastic (non-Rutherford) backscattering spectrum of painting (lead-white on canvas) with fit using roughness algorithm (data from Beck et al, ref.5, Fig.3). 